\title{
Impaired recognition of negative facial emotions in patients with frontotemporal dementia
}

\author{
Diego Fernandez-Duque ${ }^{\mathrm{a}, \mathrm{b}, *}$, Sandra E. Black ${ }^{\mathrm{b}}$ \\ ${ }^{a}$ Department of Psychology, Villanova University, 800 Lancaster Avenue, Villanova, PA 19085, USA \\ ${ }^{\mathrm{b}}$ Department of Medicine, Sunnybrook and Women's College Health Science Centre, University of Toronto, Canada
}

Received 5 January 2005; accepted 13 January 2005

Available online 16 March 2005

\begin{abstract}
Patients with behavioral variant of frontotemporal dementia (FTD) have difficulties recognizing facial emotions, a deficit that may contribute to their impaired social skills. In three experiments, we investigated the FTD deficit in recognition of facial emotions, by comparing six patients with impaired social conduct, nine Alzheimer's patients, and 10 age-matched healthy adults. Experiment 1 revealed that FTD patients were impaired in the recognition of negative facial emotions. Experiment 2 replicated these findings when participants had to determine whether two faces were expressing the same or different emotions. Experiment 3 was a control study in which participants had to discriminate whether two faces were of the same sex. In this non-emotional processing task, both patient groups performed worse than normal participants, but FTD patients performed as well as Alzheimer's patients. We conclude that FTD patients are impaired in the recognition of negative facial emotions.
\end{abstract}

(C) 2005 Elsevier Ltd. All rights reserved.

Keywords: Emotion; Face recognition; Neuropsychology; Orbitofrontal cortex

Frontotemporal lobar degeneration encompasses a heterogeneous group of dementias with varied clinical and pathological presentations. One of its clinical presentations, the behavioral variant of frontotemporal dementia (FTD), is characterized by changes in personality, impaired social skills, poor decision making, lack of empathy and lack of insight, implying injury to the orbitofrontal cortex (McKhann et al., 2001; Mychack, Rosen, \& Miller, 2001; Neary et al., 1998). ${ }^{1}$

\footnotetext{
* Corresponding author. Tel.: +1 6105196207 ; fax: +1 6105194269 .

E-mail address: diego.fernandezduque@villanova.edu

(D. Fernandez-Duque).

${ }^{1}$ Several taxonomies exist in the literature on frontotemporal dementia, and this has sometimes led to confusion. Cases such as the ones described in this article, in which personality changes are the chief initial symptom, are sometimes called 'frontal variant' of FTD, a label that highlights the contribution of orbitofrontal cortex to those symptoms (Keane et al., 2002). However, other times they are referred to as 'temporal variant', highlighting the contribution of right anterior temporal lobe structures to behavioral disinhibition (Rosen et al., 2002). Some researchers have proposed a classification based on clinical features. The cases described in this article belonged to the behavioral variant of FTD in such a classification, as opposed to the variants in which progressive language deficits are the main feature (e.g., semantic dementia, primary progressive aphasia) (McKhann et al., 2001).
}

Although it has an insidious onset and a gradual progression, FTD in this clinical presentation bears close resemblance to cases of orbitofrontal damage caused by traumatic brain injury (Rosen et al., 2002). Those patients are often impaired not only in social behavior, but also in more basic aspects of social communication, such as the ability to recognize facial emotions (Hornak, Rolls, \& Wade, 1996). Given the similarities in their impaired social behavior and in anatomical correlates between the two groups, we hypothesized that FTD patients, like patients with orbitofrontal lesions, would be impaired in the recognition of facial emotions.

Besides the clinical implications of FTD, the question of whether patients with this type of dementia are impaired in recognizing facial emotions is important for understanding the neural architecture underlying emotion and face processing. Both theoretical and empirical arguments have been gathered in support of specialized brain areas that separately recognize facial identity and facial emotion (Bruce \& Young, 1986). Thus, some prosopagnosic patients are sometimes unimpaired at recognizing facial emotions (Humphreys, Donnelly, \& Riddoch, 1993; Tranel, Damasio, \& Damasio, 1988), and patients with normal recognition of 
facial identity sometimes have difficulties recognizing emotional expressions (Anderson, Spencer, Fullbright, \& Phelps, 2000; Young et al., 1993). In functional neuroimaging studies, emotional and non-emotional facial features activate different brain areas. The structural aspects of face processing activate ventral occipitotemporal areas (Kanwisher, McDermott, \& Chun, 1997), while emotional features activate a network of limbic structures that includes the amygdala, insula, and orbitofrontal cortex (Blair, Morris, Frith, Perret, \& Dolan, 1999; Calder, Lawrence, \& Young, 2001; Phillips et al., 1997; Whalen et al., 1998). Those limbic structures are affected in FTD, while occipitotemporal areas are relatively spared (Boccardi et al., 2002; Rosen et al., 2002). Thus, it is reasonable to hypothesize that FTD patients will be impaired in the recognition of facial emotion, but not in the recognition of non-emotional facial features. On the other hand, certain brain areas that may be implicated in the recognition of facial emotion, such as somatosensory cortex, are relatively spared in FTD, raising the possibility that FTD patients may be capable of normal facial emotion recognition (Adolphs, Damasio, Tranel, Cooper, \& Damasio, 2000; Bocti, Rockel, Roy, Gao, \& Black, 2004).

The issue of specific processing of facial attributes can be taken a step further by asking whether certain emotions will be more affected than others. It is a matter of current debate whether separate brain areas represent individual emotions such as anger, fear, and disgust, or instead the brain encodes dimensions such as valence and arousal from which a space of emotional experiences arise. This debate notwithstanding, there is some evidence that the limbic structures affected in FTD are critical for the recognition of many negative emotions (Adolphs, Tranel, Damasio, \& Damasio, 1994; Blair et al., 1999; Calder et al., 2001; Harmer, Thilo, Rothwell, \& Goodwin, 2001; Hornak, Rolls, \& Wade, 1996). The social misconduct and personality changes exhibited by FTD patients also hint at the possibility of a specific impairment in the perception of emotions. Anecdotal evidence suggests that FTD patients behave as if they are unable or unwilling to make appropriate use of the social feedback conveyed in expressions of anger, sadness, fear or disgust.

The hypothesis that FTD patients will be specifically impaired in the recognition of negative emotions is complicated by the fact that even normal participants have more difficulties recognizing negative emotions than positive ones (Ekman \& Friesen, 1975; Russell, 1994). It is unclear whether negative facial emotions per se are more difficult to recognize, or instead the difference is due to a test stimulus artifact. In either case, the difference between negative and positive displays raises the possibility that task difficulty might underlie patients' poor performance. In other words, FTD patients, due to their general cognitive deficits, may be disproportionately impaired in the most difficult trials, which happen to be the ones depicting negative emotions (Rapcsak et al., 2002). We addressed this problem in two ways. First, our study included a group of Alzheimer's (AD) patients, which was matched to the FTD group for cognitive ability. If poor recognition of negative emotions stemmed from pictures of negative emotions posing a more difficult task, then both groups should be equally impaired. The inclusion of a cognitively impaired comparison group also minimized the chances of obtaining ceiling effects, which often muddle the interpretation of interactions. Second, we compared patients' recognition of 'difficult' and 'easy' negative emotions. Past literature reveals that healthy adults often err in the recognition of facial expressions of fear, but are almost flawless in the recognition of facial expressions of anger (Ekman \& Friesen, 1975; Rapcsak et al., 2002). Thus, a level-of-difficulty account would predict that FTD patients should be severely impaired in the recognition of fear (a difficult emotion to recognize) while being relatively spared in the recognition of anger (an easy emotion to recognize). An account based on a specific deficit for negative emotions would predict, instead, that both 'easy' and 'difficult' negative emotions should pose a challenge for FTD patients.

The current study builds upon previous studies of facial emotion recognition in FTD (Fernandez-Duque \& Black, 2002; Keane, Calder, Hodges, \& Young, 2002; Lavenu, Pasquier, Lebert, Petit, \& Van der Linden, 1999; Perry et al., 2001; Rosen et al., 2002). The evidence from these studies converges to suggest that the inability to recognize facial emotions in FTD is caused by an inability to recognize emotions rather than an inability to recognize facial features. In fact, recognition of non-emotional features, such as face identity, appeared to be relatively unimpaired. However, these studies did not allow a direct comparison between emotional and non-emotional tasks because different stimuli and paradigms were used. Another problem of interpretation stems from ceiling or near-ceiling performance in many of the non-emotional tasks. This raises the possibility that the emotional tasks were generally more difficult, which may explain patients' poor performance. The argument for a specific impairment in facial emotion recognition would be bolstered by increasing the difficulty of the non-emotional task, thus reducing ceiling effects, and showing group by task cross-over interactions. Our study aimed to provide such evidence.

In summary, our study investigated facial emotion recognition in patients with FTD, whether their emotion recognition deficit was most severe for negative emotions, and whether it could be accounted for by general cognitive deficits. Experiment 1 asked participants to choose the correct label for a face displaying a basic emotion. We hypothesized that the FTD group would be impaired relative to the cognitively matched AD group, that the impairment would be most severe for negative emotions, and that both 'easy' and 'difficult' negative emotions would pose a challenge for patients with FTD. Experiment 2 extended the findings to a same/different-emotion discrimination with reduced cognitive demands. Experiment 3 provided a measure of nonemotional facial processing by using a same/different sex discrimination task. Also, in Experiment 3 we explored the automatic processing of facial emotions: we hypothesized 
that performance in the sex discrimination task would be influenced by the emotion information in the healthy elderly and AD groups, but not in the FTD group. The results of the three experiments were largely consistent with our hypotheses, and together they support the view that FTD patients are selectively impaired in the recognition of negative emotions.

\section{Experiment 1}

Experiment 1 provided an initial assessment of whether patients with frontotemporal dementia are impaired in the ability to recognize facial emotions. Faces depicting emotions were displayed one at a time and participants were instructed to select the corresponding emotional label.

We also investigated some more specific questions. First, we asked whether FTD patients' poor performance could be accounted for by general cognitive deficits. For this, we compared FTD and AD groups matched for cognitive impairment. Second, we asked whether emotion recognition in FTD patients would be most impaired for expressions carrying a negative valence. To test this, we assessed participants' responses to each emotion separately. A third question, related to the previous ones, was whether poor recognition of negative emotions could be accounted for by a level-of-difficulty explanation. A level-of-difficulty explanation would predict that both patient groups should show a larger impairment to the most difficult emotions (i.e., the emotions that healthy elderly have most difficulty with). An explanation based in a selective deficit of negative emotion recognition would predict that the impairment should be of similar magnitude for easy and difficult negative emotions, and be present only in patients with FTD.

Another question we asked in experiment 1 was whether FTD patients were capable of categorizing emotions as positive and negative. For this, we looked at whether errors crossed emotional valence (e.g., a happy face labeled as sad, or an angry face labeled as happy). Finally, we explored whether the error patterns were similar across patient groups, or instead there were systematic deviations in what different groups perceived.

\subsection{Method}

\subsubsection{Participants}

Six patients with clinical diagnosis of frontotemporal dementia (FTD), nine patients with clinical diagnosis of Alzheimer's disease (AD), and ten age-matched normal participants (NCs) participated in the study. All FTD patients met Lund-Manchester Criteria (Neary et al., 1998), and all the AD patients met criteria for probable Alzheimer's disease, as established by the workgroup of the National Institute of Neurological and Communicative Disorders and Stroke-Alzheimer's Disease and Related Disorders Association (NINCDS-ADRDA) (McKhann et al., 1984). Only pa- tients with mild dementia were selected, based on a cut-off score of 20 in the Mini-Mental State Examination. ${ }^{2}$

To certify that the AD and FTD groups were matched for cognitive abilities, patients completed a neuropsychological assessment. Five normal participants were also tested and their performance was compared to the patient groups. Table 1 shows the results of the neuropsychological tests for the three groups (for a more detailed description, see Appendix A).

As expected, both patient groups were impaired relative to the normal participants in most domains. More importantly, however, there was no cognitive domain in which FTD patients were significantly worse than AD patients. The FTD group never performed more than one standard deviation below the AD group, and performance by the FTD group was indistinguishable from the AD group in visuospatial ability (Line Orientation Task) and in the recognition of unfamiliar faces (e.g., Benton Face Recognition Task).

Behavioral symptoms were assessed with the Frontal Behavioral Inventory (Kertesz, Nadkarni, Davidson, \& Thomas, 2000), the Neuropysychiatric Inventory area (Cummings et al., 1994), and the Cornell Scale for Depression in Dementia (Alexopoulos et al., 1988Alexopoulos, Abrams, Young, \& Shamoian, 1988) (for a more detailed description, see Appendix A). All six FTD patients had some signs of neuropsychiatric dysfunction, including disinhibition, aberrant motor behavior, apathy, and changes in appetite. In contrast, only two of the nine AD patients had neuropsychiatric problems. Consistent with the overlap of symptoms between FTD and depression in terms of apathy, changes in appetite, and irritability, four FTD patients had high scores in the Cornell Depression Scale. FTD patients were being treated for depressive symptoms or behavioral abnormalities with SSRIs $(N=4)$ or atypical neuroleptics $(N=2)$. No patient was psychotic nor met clinical depression criteria at time of testing.

The abnormal scores on the depression symptom scale raise the question as to whether impaired emotion recognition may be secondary to depression. However, the patterns of results found in depressed patients are opposite to the ones hypothesized for FTD patients in this study. In particular, depressed patients sometimes show a negative bias, with high accuracy for labeling sadness and relatively poor accuracy labeling happiness (Mandal and Bhattacharya, 1985Mandal \& Bhattacharya, 1985).

To rule out contributions from other pathologies, MRI was performed with a $1.5 \mathrm{~T}$ GE Signa scanner using standard protocol (Callen, Black, Gao, Caldwell, \& Szalai, 2001). Apart from atrophy consistent with their dementia, the scans showed no other pathology. Cerebral blood flow was measure in both patient groups using single-photon emission computed tomography (SPECT). Five of the six FTD patients

\footnotetext{
2 Patients were recruited primarily through the Cognitive Neurology Unit at Sunnybrook and Women's Health Sciences Centre in Toronto, where the project received approval from the Ethics Board. Consent for participation in the study was obtained from the patients and their caregivers.
} 
Table 1

Demographic, neuropsychiatric, and neuropsychological information

\begin{tabular}{|c|c|c|c|c|}
\hline & Maximum score & $\mathrm{NC}$ & $\mathrm{AD}$ & FTD \\
\hline Age & 80 & $65.1(8.4)$ & $70.1(7)$ & $63.7(6.4)$ \\
\hline Sex: male-female ratio & & $4 / 6$ & $5 / 4$ & $5 / 1$ \\
\hline Years of education & & $15.7(3.6)$ & $15.9(3.5)$ & $16.5(3.8)$ \\
\hline Frontal behavioral inventory & 72 & $\mathrm{n} / \mathrm{a}$ & $18(11)$ & $37.8(12)$ \\
\hline Neuropsychiatric inventory & 144 & $\mathrm{n} / \mathrm{a}$ & $14(18)$ & $31.6(18)$ \\
\hline Cornell scale for depression & 38 & $\mathrm{n} / \mathrm{a}$ & $9.1(6.8)$ & $13.6(8)$ \\
\hline MMSE & 30 & $29(0.7)$ & $24.8(2.0)$ & $26.5(2.3)$ \\
\hline DRS (total) & 144 & $140(1.1)$ & $125.7(9.9)$ & $125.7(6.3)$ \\
\hline Boston naming & 30 & $27.8(1.3)$ & $21.3(7.4)$ & $21.6(6.8)$ \\
\hline WAB comprehension & 10 & $9.97(.07)$ & $9.92(0.07)$ & $9.82(0.34)$ \\
\hline Verbal fluency (FAS) & & $47.9(15)$ & $29.3(15)$ & $25.2(12)$ \\
\hline Semantic fluency & & $19(6)$ & $10.3(4)$ & $13.2(5)$ \\
\hline Pyramids and Palms ${ }^{\mathrm{a}}$ & 52 & $\mathrm{n} / \mathrm{a}$ & $\mathrm{n} / \mathrm{a}$ & $48.8(3.2)$ \\
\hline CVLT acquisition $^{\mathrm{b}}$ & 80 & $46(7.7)$ & $24.2(9.7)$ & $30.8(12.9)$ \\
\hline CVLT long delay free recall & 80 & $9.2(3.4)$ & $0.9(1.5)$ & $4.4(3.4)$ \\
\hline Line orientation task & 30 & $25.6(6)$ & $22.2(5)$ & $20(9)$ \\
\hline Visual memory immediate & 41 & $32(3)$ & $16.7(5)$ & $17.7(4)$ \\
\hline Visual memory delayed & 41 & $23.8(4)$ & $2.7(3)$ & $3.4(4)$ \\
\hline Forward digit span & 12 & $9.1(1.6)$ & $9.3(1.9)$ & $8.3(2.6)$ \\
\hline Backward digit span & 12 & $7.75(1.7)$ & $6.3(2.5)$ & $5.5(2.4)$ \\
\hline Trails A & $\mathrm{n} / \mathrm{a}$ & $36.7(9)$ & $47.5(17)$ & $36.5(8)$ \\
\hline Trails $B^{c}$ & $\mathrm{n} / \mathrm{a}$ & $79.2(22)$ & $178(85)$ & $117(45)$ \\
\hline $\mathrm{B}$ to $\mathrm{A}$ ratio & $\mathrm{n} / \mathrm{a}$ & $2.2(0.4)$ & $3.8(1.9)$ & $3.3(1.3)$ \\
\hline WCST correct ${ }^{\mathrm{d}}$ & 64 & $44(9)$ & $39.6(10)$ & $45.6(11)$ \\
\hline Benton face recognition & 54 & $48.2(3.7)$ & $42.3(3)$ & $41.7(2.1)$ \\
\hline
\end{tabular}

MMSE: Mini-Mental State Examination; DRS: Dementia Rating Scale; WAB: Western Aphasia Battery; CVLT: California Verbal Learning Test; WCST: Wisconsin Card Sorting Task.

a Cut-off score for impairment is 46.8 (90\%).

b FTD case 4 completed the Hopkins Verbal Learning Test instead of the CVLT, and performed within normal limits.

c No data were collected for one AD patient, who failed to understand trails B instructions.

d No data were collected for FTD case 1, as the patient refused to complete the task.

showed frontal temporal hypoperfusion, and eight of the nine AD patients showed posterior hypoperfusion patterns consistent with AD (Neary et al., 1987).

\subsubsection{Equipment}

All the experiments were carried out on a Dell Inspiron laptop computer with Windows 98 operating system, and a 15 in. monitor, set to a screen resolution of $1024 \times 768$ pixels. Stimulus display and response collection were achieved using E-prime, a commercial experiment application. Touch responses were collected by an attachable touchscreen (Edmark Touchwindow E 1014), and relayed to the computer via a USB connector.

\subsubsection{Stimuli}

Photographs of neutral faces and the six basic emotions (sad, happy, surprised, angry, disgusted, frightened) from the Ekman and Friesen series were selected. For each emotion, we chose the seven faces that led to highest recognition levels in previously reported norms. For 'fear' and 'disgust', an eighth photograph was added after a preliminary study revealed unusual difficulties in recognizing the emotions depicted by one of the photographs in these categories (see Section 1.1.5). Each photograph was $13.5 \mathrm{~cm} \times 9 \mathrm{~cm}$ in size, had a gray background surrounded by a thin black frame, and was displayed onto the white background of the computer screen. The emotion labels were displayed in black 26 pt Courier New font, along each side of the photograph. 'Sad', 'happy', and 'surprised' appeared from top to bottom on the left side, 'disgusted', 'frightened', and 'angry' were displayed from top to bottom on the right, and 'neutral' was centered below the photograph. The labels remained on the screen during the total duration of the experiment. Each label had a response area delimited by a black rectangle, $7 \mathrm{~cm} \times 3 \mathrm{~cm}$ in size. The border of the rectangles were $2 \mathrm{~cm}$ away from the outer border of the photograph, and there was a $2.5 \mathrm{~cm}$ vertical distance between each rectangle's borders and those of its neighbors.

\subsubsection{Procedure}

Each participant completed two sessions, on separate days. ${ }^{3}$ In the initial session, participants were taught how to use the touchscreen and practiced until they reported feeling comfortable with its use. Participants were instructed that

\footnotetext{
${ }^{3}$ The face recognition tasks reported in this article (Experiments 1-3) were a subset of a larger battery which also included tasks on theory of mind, emotional understanding in short vignettes, empathic accuracy in videotaped interviews, and a set of personality questionnaires. To minimize carry-over effects, the facial recognition tasks were intermixed with other parts of the battery. The findings from those other tasks are reported elsewhere (Fernandez-Duque, Hodges, \& Black, 2005).
} 
responses inside the rectangular area would be recorded and would trigger a feedback tone. Participants had the option to report their answer by touch or verbally, in which case the experimenter entered the response via touchscreen. Participants were encouraged to make a response in every trial but accuracy was emphasized over speed. Faces were displayed one at a time and remained on the screen until response or for a maximum of $30 \mathrm{~s}$. In the rare occasions in which time expired before the participant made a response, the trial was repeated at the end of the session.

There were seven practice trials - one for each emotion which were not included in the data analysis. The same seven photographs were used as practice for all participants. For each practice trial, the experimenter read the seven labels, at a rate of $1 \mathrm{~s}^{-1}$, from top to bottom, starting on the lefthand side (happy, sad, surprised), continuing on the righthand side (disgusted, frightened, angry), and finishing on the bottom (neutral). No accuracy feedback was given during practice nor during actual testing. The only feedback that participants received, besides the auditory tone announcing that a response had been recorded, occurred in practice trials in which participants selected the 'neutral' response. In those trials, the experimenter said "Remember, we choose neutral when the face is not showing any emotion. If the face is showing no emotion, you will choose neutral. If the face is showing an emotion but you are not sure which one, you will make a guess from one of the other labels". We included this feedback because in previous pilot studies participants would sometimes choose 'neutral' to mean 'I don't know'.

During testing, the photographs were presented in random order. There were a total of 51 test trials per session (eight trials for fear, eight for disgust, and seven for each of the other emotions). At various points during the session, the experimenter would remind participants of the instructions by saying "how is s/he feeling? Is s/he..." and then reading the seven labels in the aforementioned fixed order. Participants were reminded of the instructions whenever they made several errors in a row. Participants who made few errors were reminded of the instructions approximately three times in each session.

\subsubsection{Preliminary study}

To confirm that the facial emotions in the photographs we selected were highly recognizable, we conducted a pilot study on 20 undergraduate students from University of Toronto (mean age: 20 years; S.D. $=2.7$ ). We used the same procedure described above. Percent accuracy in young adults was as follows (standard deviation in parenthesis): happy 97
(7), neutral 98.6 (4), surprised 98.6 (4), sad 89 (15), disgust 84 (20), fear 83.8 (15), angry 94 (8). Overall performance was very good at $92.1 \%$ accuracy, suggesting that the photographs we selected depicted highly recognizable emotions. However, there were two faces (one depicting fear, the other depicting disgust) that were mislabeled by more than $40 \%$ of participants. To compensate for these unusually difficult trials, we added one other photograph of fear and one other photograph of disgust to the stimuli set.

\subsection{Results}

For each participant, data from the two sessions were aggregated, and an average was calculated for each emotion. We compared performance across groups in each of the emotions (see Table 2). We report mostly non-parametric tests, which protect against violations of the normal distribution. Analyses of variance yielded comparable results to the nonparametric tests, and are reported if they provide additional information.

There were group differences for emotions of fear, anger, disgust, and surprise (Kruskal-Wallis non-parametric test, 2 d.f., $H \geq 6.4, p<0.05$ ). There was also a non-significant trend for perception of sadness (Kruskal-Wallis non-parametric test, 2 d.f., $H=4.7, p<0.09$ ). Follow-up analyses revealed that, relative to age-matched normal participants, FTD patients were impaired in the recognition of all negative emotions [anger: $U=2.5, Z=3.1, p<0.002$; disgust: $U=9$, $Z=2.3, p<0.02$; fear: $U=8.5, Z=2.3, p<0.02$; sadness: $U=13.5, Z=1.8, p<0.07]$. Relative to AD patients, FTD patients were impaired in the recognition of anger $(U=6$, $Z=2.5, p<0.01)$, disgust $(U=8.5, Z=2.2, p<0.03)$, fear $(U=2.5, Z=2.9, p<0.004)$, and surprise $(U=6.5, Z=2.5$, $p<0.01)$. No differences were found between AD patients and normal participants for any of the emotions $(p>0.10)$. To explore this question more thoroughly, data were submitted to a mixed analysis of variance that had Group (AD, NC) as a between-subjects factor and Emotion as a within-subject factor. This more powerful analysis also failed to reveal a difference between the two groups, $F(1,17)=0.001$, ns, or an interaction between emotion and group, $F(6,102)=0.8$, ns.

Could the impaired recognition of negative emotions be accounted for by a level-of-difficulty explanation? To explore this question, we selected the most difficult negative emotion (i.e., the one to which healthy subjects made the most errors) and the easiest one (i.e., the one to which healthy subjects made the fewest errors). Consistent with previous literature, these were fear and anger, respectively. Next, we asked

Table 2

Percent correct (and standard deviations) for facial emotion recognition in Experiment 1

\begin{tabular}{|c|c|c|c|c|c|c|c|c|}
\hline & Happiness & Neutral & Surprise & Sad & Disgust & Fear & Anger & Average \\
\hline $\mathrm{NC}$ & $95(5.7)$ & $88(20.2)$ & 89 (10.8) & $85(22.1)$ & $90(8.8)$ & $66(24.2)$ & $96(6.9)$ & 87 (9.6) \\
\hline $\mathrm{AD}$ & $95(6.0)$ & $92(7.9)$ & $95(7.1)$ & $78(18.0)$ & $88(7.2)$ & 70 (14.4) & $91(11.8)$ & $87(5.1)$ \\
\hline FTD & $100(0.0)$ & $75(21.2)$ & 75 (21.6) & $62(25.4)$ & $65(19.1)$ & $34(16)$ & $55(27.0)$ & $66(9.5)$ \\
\hline
\end{tabular}


whether dementia led to a disproportionate cost in recognizing the most difficult emotion (fear), relative to the easiest negative emotion (anger). The performance by the healthy elderly group served as baseline. The FTD group was 32\% below baseline in the recognition of the most difficult negative emotion (fear), and $41 \%$ below baseline in the recognition of the easiest negative emotion (anger), a pattern contrary to the level-of-difficulty hypothesis. The AD group was $4 \%$ above baseline in the recognition of the most difficult negative emotion (fear), and 5\% below baseline in the recognition of the easiest negative emotion (anger). Once again, this pattern is contrary to the level-of-difficulty hypothesis.

To explore the level-of-difficulty hypothesis more systematically, we ran a mixed analysis of variance with Group (NC, $\mathrm{AD}, \mathrm{FTD}$ ) as a between-subjects factor and Emotion (fear, anger) as a within-subject factor. As expected, this analysis revealed main effects of Group, $F(2,22)=16, p<0.001$, and Emotion, $F(1,22)=28, p<0.001$. Most importantly, the two effects were additive, with no significant interaction, $F(2,22)=0.4$, ns. This argues against a level-of-difficulty interpretation. We also tested the FTD group against chance performance for fear recognition $(14.3 \%)$, to rule out a possible bias brought about by near-floor performance. Although the FTD group was severely impaired in fear recognition, these patient group did perform better than chance, $t(5)=3, p<0.03$.

Next, we examined individual scores to assess how many patients were impaired. Almost none of the AD patients fell in the lowest 5th percentile of the distribution for any of the emotions (see Table 3). In contrast, all the FTD patients were impaired in recognizing at least one negative emotion. Even case 3, who performed within normal limits on most emotions, was impaired in the recognition of one negative emotion (fear). FTD patients only seldom were impaired in positive (happy) and non-negative (neutral, surprise) emotions. Five out of 6 FTD patients were impaired in recognizing 'easy' negative emotions, such as anger and disgust, a result that again argues against a level-of-difficulty interpretation.

In another approach to the data, we explored the error patterns for systematic variations. This exploratory analysis is most revealing for emotions with a sizeable number of errors. For this reason, we limited the analysis to faces depicting negative emotions. Error responses were not randomly distributed. For example, negative emotions almost never triggered a happy response $(0.5 \%)$. The error rates also exhibited other, more specific, patterns. Emotions of disgust and anger were often confused with each other, as were the emotions of fear and surprise, and sad faces were often confused with neutral expressions. These patterns of errors were very similar to those reported in previous studies with normal adults (Anderson et al., 2000; Rapcsak et al., 2002). Surprise faces were mislabeled as fear but also as happy, revealing the ambiguous valence of this emotion. Error patterns were largely the same for FTD and the comparison groups. FTD patients followed the comparison groups in their tendency to confuse disgust and anger, sadness and neutral, and to mislabel fear as surprise. One exception to this trend was the disproportionate tendency, by FTD patients, to label angry faces as being sad.

\subsection{Discussion}

Experiment 1 revealed that, relative to normal participants and Alzheimer's patients, FTD patients were impaired in the ability to recognize facial emotions. This impairment was most pronounced in the recognition of negative emotions. In contrast, FTD patients were as good as the comparison groups in the recognition of happy faces, and almost never did they mislabel a negative emotion as 'happy'. These results suggest that FTD patients were capable of valence discrimination (i.e., is this emotion positive or negative?), but had difficulties making subtler discriminations from the pool of negative emotions. FTD patients were impaired in the recognition of negative emotions independent of whether those emotions were 'easy' (anger, disgust) or 'difficult' (fear) to recognize by normal subjects. This pattern argues against a level-of-difficulty interpretation, and points instead toward an specific deficit in processing negative emotions.

The findings from Experiment 1 also rule out the possibility that the impaired performance by the FTD group was due to general cognitive deficits. The AD group, which had general cognitive deficits as large as the FTD group, performed significantly better than the FTD group in facial emotion recognition. In fact, the $\mathrm{AD}$ group performed as well as the normal participants. This latter finding may, at first sight,

Table 3

Individual data from patients with frontotemporal dementia in Experiment 1, and number of patients in the lowest 5th percentile of the normal distribution

\begin{tabular}{|c|c|c|c|c|c|c|c|c|}
\hline & Happiness & Neutral & Surprise & Sad & Disgust & Fear & Anger & Average \\
\hline$\overline{1}$ & 100 & 64.5 & 86 & 64.5 & $44^{\mathrm{a}}$ & 62.5 & $71^{a}$ & $70.3^{b}$ \\
\hline 2 & 100 & $39.5^{\mathrm{a}}$ & $37^{\mathrm{a}}$ & $28.5^{\mathrm{a}}$ & $62.5^{\mathrm{a}}$ & 31.5 & $64.5^{\mathrm{a}}$ & $51.9^{\mathrm{a}}$ \\
\hline 3 & 100 & 86 & 78.5 & 78.5 & 100 & $21.5^{\mathrm{b}}$ & 93 & 79.6 \\
\hline 4 & 100 & 71 & 93 & 100 & $62.5^{\mathrm{a}}$ & $19^{\mathrm{b}}$ & $28.5^{\mathrm{a}}$ & $67.7^{\mathrm{b}}$ \\
\hline 5 & 100 & 86 & 93 & 57 & $69^{\mathrm{a}}$ & 27 & $50^{\mathrm{a}}$ & $68.8^{\mathrm{b}}$ \\
\hline 6 & 100 & 100 & $64^{\mathrm{b}}$ & $43^{\mathrm{b}}$ & $53.5^{\mathrm{a}}$ & 40.5 & $21.5^{\mathrm{a}}$ & $60.3^{\mathrm{a}}$ \\
\hline \multicolumn{9}{|c|}{ Below 5 th percentile } \\
\hline FTDs $(n=6)$ & 0 & 1 & 2 & 2 & 5 & 2 & 5 & 5 \\
\hline $\operatorname{ADs}(n=9)$ & 0 & 0 & 0 & 1 & 0 & 0 & 1 & 0 \\
\hline
\end{tabular}

${ }^{\text {a }}$ Scores below the 1 st percentile of the normal distribution (i.e., S.D. $<-2.33$ ).

b Scores below the 5 th percentile. 
seem to be a departure from previous studies showing AD impairment in emotion recognition (Albert, Cohen, \& Koff, 1991). However, the range of cognitive impairment in those previous studies was much larger than in ours. Furthermore, the poor performance was accounted for by impaired cognitive ability rather than by a specific impairment in facial emotion recognition. Thus, Experiment 1 is broadly consistent with those findings, in that it showed that AD patients with mild cognitive deficits were not impaired in emotion recognition.

When FTD patients made an error, their choices were very similar to the choices made by the comparison groups. For example, expressions of fear were mistaken to be expressions of surprise in all three groups. Thus, although FTD patients were impaired in their ability to recognize negative emotions, their perception seemed qualitatively similar to that of participants in the comparison groups. One exception was the perception of angry faces, which FTD patients, unlike other groups, often perceived as an expression of sadness.

\section{Experiment 2}

Experiment 2 aimed to replicate the findings of Experiment 1 and to further explore the factors contributing to FTD patients' difficulties in recognizing negative emotions. Two faces were displayed side-by-side and participants reported whether the pair of faces depicted the same or different emotions.

The design of Experiment 2 reduced some of the general cognitive demands of Experiment 1 by reducing the number of alternatives and eliminating the use of verbal emotion labels. These modifications also rectified another limitation of Experiment 1, namely the fixed location of emotion labels in the computer screen, which might have contributed to response biases. To control for differences in speed-accuracy criterion, the design of Experiment 2 kept the display exposure constant at $1500 \mathrm{~ms}$, instructed participants to 'go with the flow and rely on first impressions', and recorded response times.

Although the goal of Experiment 2 was to explore emotional facial processing, it was important that the stimuli and design be applicable also to a non-emotional facial sex discrimination task (Experiment 3), so that a direct comparison between emotional and non-emotional facial processing could be drawn. To meet these demands, faces included only internal features so that sex information could not be extracted from hairstyle or ear accessories. Moreover, sex similarity and emotion similarity were balanced so that the probability of 'same emotion' trials was independent of sex similarity. Finally, an equal number of same sex and different sex trials were presented. These aspects of the design, although irrelevant for Experiment 2 (i.e., emotion recognition task), will become critical in Experiment 3 (i.e., sex recognition task).

\subsection{Method}

\subsubsection{Participants}

Participants were the same as in experiment 1, with the exception of one $\mathrm{AD}$ patient who was not available to complete this experiment.

\subsubsection{Stimuli}

Photographs of the six basic emotions (sad, happy, surprised, angry, disgusted, frightened) from the Ekman and Friesen series were selected. Unlike Experiment 1, we did not include neutral faces. The photographs were modified using Adobe Photoshop to add a gray oval filter that completely masked the external facial features. The size of each face was $8 \mathrm{~cm} \times 5.2 \mathrm{~cm}$, and faces were displayed $3 \mathrm{~cm}$ apart from each other. The photographs were displayed against a gray computer background.

The photographs were grouped into pairs, of which half showed the same emotion (e.g., happy-happy) and half showed different emotions (e.g., sad-happy). Half of the pairs depicted faces of the same sex-but never the same identity - and the other half depicted faces of different sex. These two factors (sex similarity, emotion similarity) were balanced.

There were 28 trials in which the emotion depicted was the same for the two faces. Sixteen of these trials depicted a negative emotion (four trials for each emotion), and the other 12 trials depicted an emotion that was not negative (six happy and six surprise). This was a compromise between having an equal number of negative and non-negative trials, and having an equal number of trials for each emotion. The other 28 trials depicted faces with different emotions. In 12 of these trials, one of the emotions was positive (happy) and the other was negative (sad, fear, anger, disgust), and in the remaining 16 trials both emotions were negative (e.g., fear and disgust). Each emotion was depicted on the left and right sides of the screen with close to equal probability.

In selecting pairs of faces with the same emotion, we tried to minimize their superficial similarity. This is difficult to achieve, because facial expressions have a correspondence with superficial (i.e., observable) facial features. Thus, two faces showing the same emotion are bound to look more similar than two faces expressing different emotions. Nonetheless, a certain amount of variability exists in the ways that an emotion can be expressed, and this variability can be used to minimize the feature similarities. For example, anger can be expressed by an open mouth with teeth showing, but it can also be expressed by a closed mouth with tight lips. We used that variability when pairing faces of the same emotion, as a way to discourage a strategy based on simple feature matching. By the same logic, we tried to maximize the feature similarity in pairs that depicted two different emotions.

\subsubsection{Procedure}

Two faces were displayed simultaneously $3 \mathrm{~cm}$ apart from each other and remained on the screen for $1500 \mathrm{~ms}$, after 
which they were replaced by a small circle in the center of the screen. The circle remained for $9 \mathrm{~s}$, or until the participant made a verbal response, at which point it was replaced by a small cross to signify that a response had been recorded. Verbal onset response was measured relative to the onset of the faces, via a serial response box (model 200a) attached to the computer. Following their verbal response, the experimenter entered the participants' answer by pressing a key in a separate keyboard via an USB connector. An interval of $2500 \mathrm{~ms}$ followed, after which a new pair of faces was displayed. There were 56 trials in the actual test. Participants were encouraged to answer correctly but also quickly. They were told that 'first impressions are as good as any', and that they should 'go with the flow and if unsure, make their best guess'.

Before starting the session, the following instructions were given: "You will see two faces on the screen. Report whether the faces are showing the same emotion or different emotions. For example, if you see two people who are sad, you would say 'Same' but if you see a person who is sad and another who is happy you would say 'Different"'. Next, participants saw four practice trials, two depicting the same emotion, and two depicting different emotions. The pairs used for practice were not included in the data analysis nor in the actual test. The practice trials were selected to be very easy, and participants were given accuracy feedback. After a correct response, the experimenter said "That's right, they are both [depicted emotion], they are showing the same emotion" or "that's right, she is [emotion A] and he is [emotion B]; they are showing different emotions". If the participant made an error, the beginning of the sentence ("That's right ...") was replaced by "Actually...". After the practice, the instructions were repeated once again. Participants were given no feedback during the actual test. At various points during the session, the experimenter would remind participants of the instructions by saying, "Are these two people showing the same emotion or different emotions?" The experimenter offered these reminders about three times in the course of the session, or any time after the participant made several errors in a row.

\subsection{Results}

\subsubsection{Accuracy}

A preliminary analysis included group (NC, AD, FTD) as a between-subjects factor, and emotion similarity (same, different) as a within-subject factor. This analysis revealed a tendency to report that both pictures were showing the same emotion: performance was worse on trials with different emotions than in trials with the same emotion, $F(1,21)=12.8$, $p<0.002$. Thus, trials depicting the same emotion were analyzed separately from trials depicting different emotions. Another reason to analyze these two types of trials separately was that, while performance in 'same' trials depended on the recognition of one emotion, performance in 'different' trials was also dependent on the processing of a second emotion.
Trials depicting the same emotion were categorized according to their emotional valence as 'positive' or 'negative'. A mixed analysis of variance included group (NC, AD, FTD) as a between-subjects factor and valence (positive, negative) as a within-subject factor. This analysis revealed a main effect of group, $F(2,21)=12.9, p<0.001$, but no effect of valence, nor an interaction between valence and group. Post hoc comparisons revealed that the FTD group was impaired relative to the $\mathrm{AD}$ group, and that both patient groups were impaired relative to the normal comparison group (Tukey HSD, $p<0.05)$. The absence of a valence effect on trials depicting the same emotion does not rule out the possibility that negative emotions could be more difficult to recognize than positive emotions. Given that observers had an overall tendency to respond 'same', the trials with same emotion are less informative than the ones with different emotions. Similarly, the absence of group differences in how valence affects performance in the 'same' trials is less informative than the analysis of possible group effects in the trials with different emotions.

Data from trials with different emotions were entered in a mixed analysis of variance that had group (NC, AD, FTD) as a between-subjects factor and valence ('positive-negative', 'negative-negative') as a within-subject factor. The analysis revealed a main effect of group, $F(2,21)=12.7, p<0.001$, and post-hoc comparisons revealed that the FTD group was impaired relative to each of the comparison groups $(p<0.05)$. The main analysis also revealed a valence main effect, as two negative emotions were more difficult to discriminate than a pairing of one negative and one positive emotion, $F(1,21)=68.1, p<0.001$. This valence effect interacted with group, $F(2,21)=5.9, p<0.01$. Although all groups had more difficulty in discriminating two negative emotions than in discriminating a negative from a positive emotion, follow-up analyses revealed that it was the FTD group that was particularly impaired in the discrimination of negative emotions. Relative to normal participants, FTD patients were significantly worse at discriminating pairs of negative emotions than pairs combining positive and negative, $F(1,14)=18.1$, $p<0.001$. A similar trend was obtained for FTD patients relative to AD patients, $F(1,12)=3.5, p<0.08$ (Table 4).

Individual data provided further support to the claim that FTD patients were impaired in their ability to discriminate emotions, and that this deficit was most pronounced for negative emotions. All six FTD patients were in the lowest 5th percentile of the normal distribution for negative emotions, and half of them were in the lowest 5 th percentile for pairs combining a positive and a negative emotion. In contrast, only one of the $\mathrm{AD}$ patients was in the 5th percentile for negative emotions and none were in the lowest 5 th percentile for pairs combining a positive and a negative emotion.

\subsection{2. $R T$}

Error trials were excluded from the RT data. We also excluded the $1.7 \%$ of correct trials that were anticipatory responses (RT less than $100 \mathrm{~ms}$ ) or extreme outliers (RT longer 
Table 4

Percent correct (and standard deviations) for Experiments 2

\begin{tabular}{llllll}
\hline Group & \multicolumn{2}{l}{ Same-emotion } & & \multicolumn{2}{l}{ Different-emotions } \\
\cline { 2 - 3 } & Positive & Negative & & Positive-negative & Negative-negative \\
\hline NC & $95(6)$ & $97(3)$ & $98(4)$ & $83(20)$ \\
AD & $94(6)$ & $87(10)$ & $99(3)$ & $70(23)$ \\
FTD & $78(12)$ & $82(12)$ & $88(5)$ & $40(5)$ \\
\hline
\end{tabular}

than $9 \mathrm{~s}$ ). For each of the conditions of interest, median response times were computed. Again, responses from trials depicting the same emotion were analyzed separately from trials depicting different emotions.

Data from trials depicting the same emotion were entered into a $3 \times 2$ mixed analysis of variance that had group $(\mathrm{NC}$, $\mathrm{AD}, \mathrm{FTD}$ ) as a between-subjects factor and valence (positive, negative) as a within-subject factor. This analysis revealed a main group difference, $F(2,21)=3.5, p<0.05$. Follow-up analyses of variance comparing each group pair revealed that normal participants were faster than both of the patient groups $\left[M_{\mathrm{NC}}=1334(\right.$ S.D. $=77) ; M_{\mathrm{AD}}=1556(\mathrm{SD}=87)$; $M_{\mathrm{FTD}}=1643$ (S.D. $\left.=100\right)$; comparison against AD: $F(1$, $16)=5.8, p<0.03$; comparison against FTD: $F(1,14)=5.6$, $p<0.03$. Most importantly, there was no difference between the patient groups, with $\mathrm{AD}$ patients responding as quickly as FTD patients.

Data from trials depicting two different emotions were similarly submitted to a $3 \times 2$ mixed analysis of variance that had group as a between-subjects factor and valence ('positive-negative', 'negative-negative') as a within-subject factor. There was a main valence effect, $F(1,21)=27$, $p<0.0001$. Responses to pairs depicting two negative emotions took longer than responses to trials pairing a negative emotion and a positive one ['Negative-Negative': $M=1746$ (S.D. =59); 'Positive-Negative': $M=1482$ (SD = 46)]. There was also a main group effect $\left[M_{\mathrm{NC}}=1328\right.$ (S.D. $\left.=64\right)$; $M_{\mathrm{AD}}=1697 \quad$ (S.D. $\left.=72\right) ; \quad M_{\mathrm{FTD}}=1717 \quad$ (S.D. $\left.=89\right) ; \quad F(2$, $21)=4.6, p<0.02]$. Follow-up analyses of variance revealed that normal subjects were faster than both patient groups [comparison against AD: $F(1,16)=7.7, p<0.01$; comparison against FTD: $F(1,14)=6.7, p<0.02]$. Most importantly, there were no differences in response time between the two patient groups.

\subsection{Discussion}

The findings from Experiment 2 again revealed emotion recognition impairment in the FTD patients, relative to $A D$ patients and normal participants. Importantly, however, this deficit was not an artifact of a speed/accuracy trade-off. FTD patients took as much time to respond as did AD patients.

In all groups, responses were slower and accuracy rates were lower for trials with two negative emotions than for trials in which a negative emotion was paired with a happy face. Thus, participants had more difficulty discriminating different negative emotions (within-valence trials) than dis- criminating emotions of opposite valence (cross-valence trials). This difference was most pronounced in FTD patients. A possible explanation of these results is that FTD patients are specifically impaired in the processing of negative emotions. An alternative explanation is that valence is an important cue for emotion discrimination, and that the ability to use this cue is relatively spared in FTD patients. This explanation is consistent with a level-of-difficulty interpretation. According to this view, FTD patients are disproportionately impaired in the discrimination of two negative emotions because this is a more difficult task than discriminating emotions of different valence. $^{4}$

\section{Experiment 3}

Experiment 2 provided converging evidence of impaired emotion recognition of negative emotions in FTD patients. In Experiment 3, the same stimuli and a similar design were used to test the processing of non-emotional attributes. Participants were instructed to report whether two faces belonged to people of the same sex or different sex. Poor performance in this sex discrimination task would suggest that FTD patients have a general deficit in face processing, while good performance would favor a more specific deficit, limited to emotional information.

Experiment 3 also provided an opportunity to explore the automatic (i.e., obligatory) processing of emotional information. More specifically, we asked whether observers would exhibit a cost when the emotion information was incongruent with the sex information. Incongruent information occurred in trials in which two faces of the same sex displayed different emotions, and in trials in which two faces of different sex displayed the same emotion. Congruent trials included the pairing of faces of same sex and same emotion, and the pairing of faces of different sex and different emotion.

To minimize the risk of participants forgetting the instructions and switching to an emotion similarity judgment, a practice session of a sex discrimination task was administered immediately before the main task. In this practice session of 26

\footnotetext{
${ }^{4}$ A direct test of the level-of-difficulty interpretation would require two conditions of comparable difficulty, one with negative emotions pairs and the other with non-negative emotion pairs (e.g., happy/surprise). Results from our lab reveal that in such a task, FTD patients are specifically impaired in the discrimination of negative emotions, a result that argues against a level of difficulty interpretation (Fernandez-Duque \& Black, unpublished data).
} 
trials, only neutral faces were displayed, forcing participants to make their judgment based on sex.

\subsection{Method}

\subsubsection{Participants}

Participants were the same as in Experiment 1.

\subsubsection{Stimuli}

Identical to Experiment 2.

\subsubsection{Procedure}

The procedure was identical to Experiment 2, except that participants responded whether the faces belonged to people of the same or different sex. The instructions warned participants that the faces would be expressing emotions, but reassured them that this was an incidental aspect of the task that they had to ignore. In particular, participants were told: "Now you are going to continue doing the same task that you have been doing so far [i.e., the practice block]. Namely, you will see a pair of faces, and have to decide whether they are of the same sex or different sex. For example, if you see two women, you will say 'same'; if you see two men, you will say 'same'; but if you see a woman and a man, you will say 'different'. Sometimes people might be smiling or frowning, but that is not important here. All you have to do is tell me whether they are of the same or different sex". As in previous experiments, verbal onset response was measured relative to the onset of the faces, via a PST serial response box (model 200a) attached to the computer. Due to a technical error, RT data for one FTD patient (case 6) were not collected.

\subsection{Results}

\subsubsection{Accuracy}

A mixed analysis of variance was conducted on the accuracy data, with group (NC, AD, FTD) as a between-subjects factor, and emotion/sex congruency (congruent, incongruent) as a within-subject factor. ${ }^{5}$ Performance was more accurate when emotion and sex provided congruent information than when they did not, $F(1,22)=5.9, p<0.02$. There was also a group main effect, $F(2,22)=4.2 p<0.03$. Post hoc comparisons revealed that normal participants were more accurate than AD patients (Tukey HSD, $p<0.05$ ). More importantly, however, there was no significant difference in accuracy of performance between the patient groups. The individual data tell a similar story: the proportion of patients performing in the lowest 5 th percentile of the normal distribution was the same for the FTD and the AD groups (33\%).

The congruency effect indicated that emotion information was being processed despite its irrelevance to the task. To

\footnotetext{
${ }^{5}$ Whether the pairs depicted faces of the same sex or different sex was not included as a factor because a preliminary analysis revealed it had no significant effect nor did it interact with other factors.
}

Table 5

Percent correct (and standard deviations) for sex discrimination in Experiments 3

\begin{tabular}{llll}
\hline & Congruent $(\%)$ & Incongruent $(\%)$ & Congruency effect $(\%)$ \\
\hline NC & $94(6)$ & $87(11)$ & 7 \\
AD & $82(6)$ & $77(8)$ & 5 \\
FTD & $84(9)$ & $83(14)$ & 1 \\
\hline
\end{tabular}

explore this effect in more detail, we ran paired $t$-tests comparing congruent and incongruent conditions in each group. Normal participants performed worse in incongruent trials than congruent ones, $t(9)=2.4, p<0.04$. In contrast, no such difference was found for FTD patients, for whom the performance in incongruent trials was almost as good as congruent ones (see Table 5). As expected, AD patients showed the same pattern of results as healthy elderly, although this congruency effect in that group failed to reach significance, $t(8)=1.5, p<0.17$.

\subsection{2. $R T$}

Next, we assessed group differences in the speed of response. Error trials were excluded from the RT data. We also excluded $0.9 \%$ of correct trials that were anticipatory responses (RT less than $100 \mathrm{~ms}$ ) or extreme outliers (RT longer than $9 \mathrm{~s}$ ). Median response times for the conditions of interest were computed. The data were submitted to a mixed analysis of variance that had group (NC, AD, FTD) as a between-subjects factor and emotion/sex congruency (congruent, incongruent) as a within-subject factor. This analysis revealed a congruency effect $\left(M_{\mathrm{CG}}=1539, M_{\mathrm{INCG}}=1619\right.$; $F(1,21)=10, p<0.004$. There was also a main effect of group, $F(2,21)=6.5, p<0.01$. Post hoc comparisons revealed that normal participants were faster than each of the patient groups (Tukey HSD, $p<0.05$ ). Most importantly, there was no difference between the FTD and the AD groups $\left[M_{\mathrm{NC}}=1299(\right.$ S.D. $=180) ; M_{\mathrm{AD}}=1753($ S.D. $=386)$; $M_{\mathrm{FTD}}=1685$ (S.D. $\left.\left.=256\right)\right]$.

\subsection{Discussion}

Both AD and FTD patients performed below ceiling, and significantly worse than healthy elderly subjects, in the sex discrimination task of Experiment 3. Thus, the task was sufficiently difficult, and its dependent variables sufficiently sensitive, to reveal differences among groups. Despite such task sensitivity, the FTD group responded as accurately and as fast as the $\mathrm{AD}$ group in the sex discrimination task. This suggests that FTD deficits in Experiment 1 or 2 were not due to a general deficit in face processing, but rather to a more specific deficit in emotional processing.

Experiment 3 also assessed the obligatory processing of emotional information, and its possible disruption in FTD. Normal participants were unable to ignore incidental emotion information, and exhibited a cost when the emotion information conflicted with the sex information. AD patients exhibited the same pattern of results as normal participants, 
although the effect failed to reach significance. More importantly, FTD patients suffered no cost when emotion information conflicted with sex information. This result indicates a failure in the automatic processing of facial emotion by patients with FTD. This finding is particularly revealing because, unlike the measures of Experiments 1 and 2, the incongruency cost is an indirect measure of emotional processing, and as such it is less susceptible to contamination by strategy.

\section{Experiments 2 and 3 joint analysis}

The findings from Experiments 2 and 3 argue for a selective impairment in the recognition of facial emotions by FTD patients. To directly test this conclusion, we submitted the data from Experiments 2 and 3 to an analysis of variance that included patient group (FTD, AD) as a between-subjects factor, and task (emotion discrimination, sex discrimination) as the within subject factor. Data from the AD patient who participated only in the sex discrimination task were excluded from this analysis.

This analysis revealed an interaction between type of task and group, $F(1,12)=13, p<0.003$. Follow-up analyses revealed that FTD patients performed worse than AD patients in the emotion recognition task, $M_{\mathrm{AD}}=87 \%$ (S.D. $=5$ ); $M_{\mathrm{FTD}}=71.9 \%($ S.D. $=5), t(12)=5.7, p<0.0001$. In contrast, there was no significant group difference in the sex discrimination task, $M_{\mathrm{AD}}=78.3 \%$ (S.D. $\left.=5\right) ; M_{\mathrm{FTD}}=83.8 \%$ $($ S.D. $=11), t(12)=1.2$, ns (Fig. 1).

The combined analysis of Experiments 2 and 3 provide strong support for the claim that FTD patients' impairment in facial recognition is limited to emotional features, and could not be accounted for by a different level of difficulty across tasks. FTD patients performed worse than AD patients in emotion discrimination, despite performing as well or better than those participants in sex discrimination.

\section{General discussion}

The findings from three experiments support the claim that frontotemporal dementia (FTD) patients are impaired in the recognition of negative facial expressions. In Experiment 1, FTD patients were impaired in the recognition of negative facial emotions, while AD patients with similar cognitive deficits performed normally. FTD patients were impaired in the recognition of 'difficult' as well as 'easy' negative emotions, arguing for a specific deficit in the processing of negative emotion, and against a levels-of-difficulty interpretation. The error pattern suggested that FTD patients were able to recognize happiness, and discriminate positive and negative expressions, but had difficulties identifying specific negative emotions. In Experiment 2, despite reduced task demands, FTD patients continued to have difficulties discriminating pairs of faces with negative emotions. However, when asked to discriminate a pair of faces based on sex rather than emotion, FTD patients performed as well as AD patients (Experiment 3). Thus, the deficit in the first two experiments was specific to emotional information of faces, particularly those of negative valence.

Our experiments suggest that frontotemporal dementia impairs the ability to recognize emotions. Before accepting this conclusion, however, at least two alternative explanations need to be ruled out. First, some patients in the current study had abnormal scores on a depression symptom scale, raising the question of whether impaired emotion recognition was secondary to depression. However, the literature on emotion recognition in depression provides no support for the

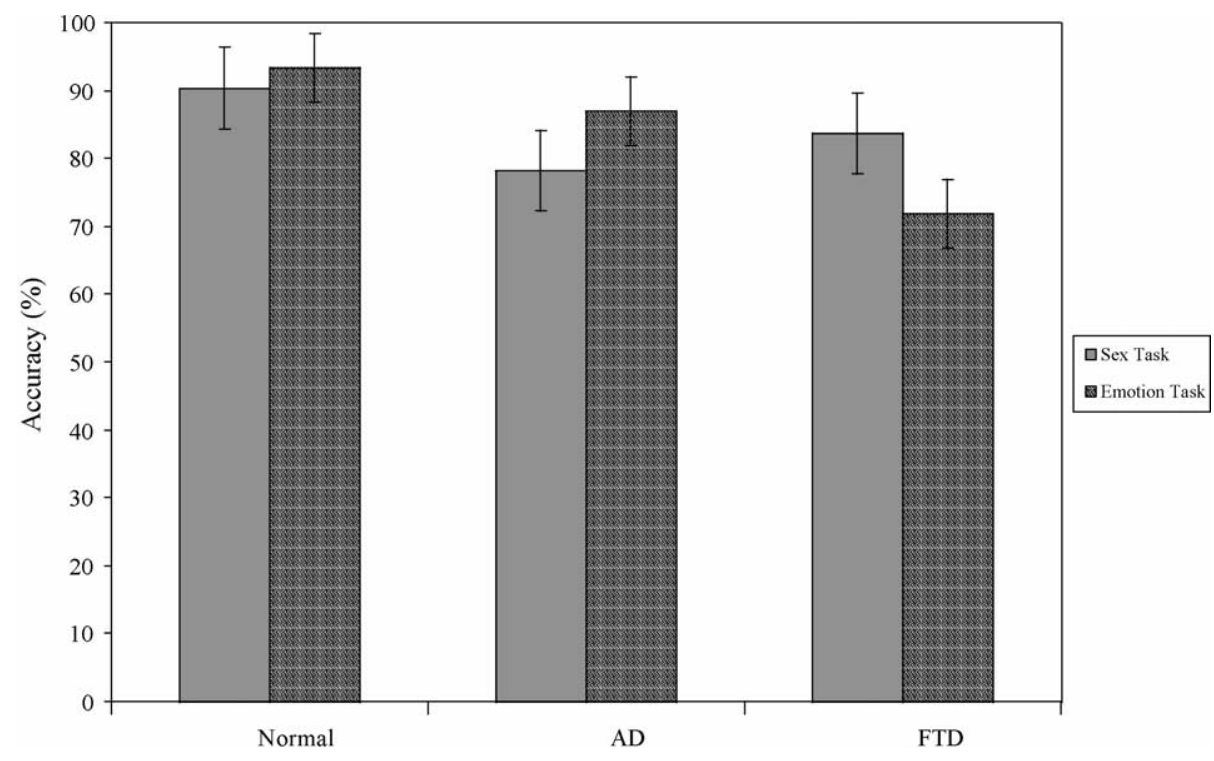

Fig. 1. Overall accuracy rates ( \pm 1 S.D.) for same/different discrimination based on sex (Experiment 3) or emotion information (Experiment 2). FTD patients were impaired in the emotion discrimination task despite performing comparable to AD patients in the sex discrimination task. 
contention that depressive symptoms could account for the pattern of results exhibited by FTD patients in these studies. Depressed patients have sometimes been described as having a negative bias, with high accuracy for labeling sadness and relatively poor accuracy labeling happiness (Mandal and Bhattacharya, 1985Mandal \& Bhattacharya, 1985). Other studies have pointed to an overall reduction in performance, both for emotional and non-emotional recognition tasks (Asthana et al., 1998). Depressed patients have sometimes been found to show normal emotion recognition in paradigms similar to our Experiment 1 (Gaebel \& Wolwer, 1992; Gessler, Cutting, Frith, \& Weinman, 1989). None of these patterns is consistent with the pattern exhibited by FTD patients, who exhibit impaired recognition of negative emotions, including some that are easily recognizable by other patient populations (e.g., anger).

A second alternative interpretation is that FTD patients' poor performance in emotion recognition was secondary to a general decrease in cognitive ability. However, the pattern of results is inconsistent with this interpretation. Although neuropsychological tests revealed the FTD group to be cognitively impaired, a group of AD patients equally impaired in cognitive tasks was able to out-perform the FTD group, frequently reaching normal performance (e.g., Experiment 1). This suggests that FTD patients' deficit was specific, and not attributable to a general cognitive loss. Further evidence that impaired performance by FTD patients cannot be explained as a general impairment in the processing of facial stimuli came from Experiment 3. In that experiment, FTD patients performed as well as AD patients in a sex discrimination task. This suggests that FTD patients are capable of processing non-emotional attributes of faces. Importantly, performance in this task failed to reach ceiling levels of accuracy. In other words, the absence of group differences cannot be attributed to a lack of test sensitivity.

The behavioral dissociation between emotional and nonemotional processing of facial features also correlates with the pattern of neuroanatomical involvement. In particular, FTD spares the face fusiform area in the temporo-occipital cortex, a region that responds selectively to faces, and that is damaged in prosopagnosic patients (Damasio, Damasio, \& Van Hoesen, 1982; Kanwisher et al., 1997). In contrast, FTD atrophy is usually evident in limbic and orbitofrontal areas, regions known to participate in many aspects of emotion regulation.

The issue of specific processing of facial attributes can be taken a step further by asking whether certain emotions are more affected than others. Our results demonstrate that patients with FTD are specifically impaired in the recognition of negative emotions. Patients with FTD were impaired not only in recognizing negative emotions that are normally difficult to identify, such as fear, but also in the recognition of negative emotions that are easily identified by healthy subjects, such as anger. FTD patients' poor performance in response to easy-to-identify negative emotions favors a true deficit in the processing of negative emotions, rather than an expla- nation based on different levels of difficulty. Future studies should address whether normal recognition of happiness by FTD patients generalizes to subtle displays of happiness in which off-ceiling performance can be measured.

Although some of the brain regions implicated in the processing of negative emotions are often dysfunctional in FTD, it would be a mistake to draw strong conclusions from our findings about the specific localization of emotions. The existence of specific anatomical substrates for individual emotions is a matter of debate (Calder et al., 2001; Rapcsak et al., 2002), and FTD is a progressive disease affecting mainly the frontal and anterior temporal regions (Bocti et al., 2004). FTD impairment in emotion recognition is likely to be caused by atrophy in orbitofrontal cortex, insula, and amygdala, and our study cannot address the unique contribution of these areas.

In other studies, FTD impairment for negative emotions was correlated with right orbitofrontal and amygdalar atrophy (Rosen et al., 2002). The right hemisphere bias is consistent with findings from the stroke literature, which point to a preferred role of the right hemisphere in emotion processing (Anderson et al., 2000; Bowers, Blonder, Feinberg, \& Heilman, 1991). The orbitofrontal and amygdalar atrophy is consistent with the role these areas play in emotion recognition (Blair et al., 1999; Hornak et al., 1996; Young et al., 1993). Nonetheless, amygdalar atrophy also occurs in AD patients (Callen et al., 2001), a group that performed close to normal in our study. Interestingly, the pattern of amygdalar atrophy appears to be different in the two diseases. FTD affects mostly the basolateral complex (Tsuchiya et al., 1999), which in the monkey has neurons that respond selectively to faces, and therefore is thought to be important for emotion recognition. Instead, AD affects mostly the corticomedial nuclei, which are phylogenetically older and modulate autonomic functions such as respiratory and cardiovascular control (Herzog \& Kemper, 1980; Hooper \& Vogel, 1976; LeDoux, 1996; Tsuchiya, \& Kosaka, 1990). Thus, different patterns of amygdalar atrophy might explain why there is poor emotion recognition in FTD but not in AD.

Another area important for face processing is the superior temporal gyrus, a region that is moderately involved in FTD (Rosen et al., 2002), and has rich connections with the amygdala and the orbitofrontal cortex (Rolls, 1999). Recognition of eye gaze direction, biological motion, and other social cues depends on the normal functioning of the superior temporal sulcus (Allison, Puce, \& McCarthy, 2000). Little is known about the abilities of FTD patients in these domains, but considering the clinical presentation of the disease, deficits are likely to exist. Such deficits, if found, could help explain the poor social skills exhibited by FTD patients. Similarly, our finding that the recognition of certain facial emotions is impaired in FTD may contribute to their socially inappropriate behavior. Faces convey information about people's feelings, as well as their reactions to the social behavior of others. Thus, an inability to recognize certain emotions may underlie in part deficits in empathy and decision making, problems that are so frequently encountered in FTD (Neary et al., 1998). At the 
same time, impaired recognition of facial emotion sometimes occurs in the absence of socially inappropriate behavior, a result that hints at a certain level of redundancy in the system. Complex social abilities are bound to draw on a multitude of cognitive and emotive functions. The job ahead of us is to uncover how such basic functions give rise to socially savvy individuals. Emotion recognition may be a first step, but in all certainty it will not be the last.

\section{Acknowledgements}

We would like to thank the patients and their families for their time and effort. We also thank Morris Freedman for referring one of his patients, Fu Qiang Gao for assistance with the neuroimaging data, and Jodie Baird for comments on an earlier draft of this paper. This research was supported by a grant from the Center for Consciousness Studies of the University of Arizona to the first author, and an operating grant from the Canadian Institute for Health Research to the second author (grant no. 13129). Diego Fernandez-Duque was supported by fellowships from the Heart and Stroke Foundation of Ontario (grant no. F 4866), the Rotman Research Institute, Baycrest Centre for Geriatric Care, and the Cognitive Neurology Unit, Sunnybrook and Women's, University of Toronto.

\section{Appendix A. Cognitive and neuropsychiatric testing}

Overall performance was assessed with the Mini-Mental State Examination (MMSE) (Folstein, Folstein, \& McHugh, 1975) and the Dementia Rating Scale (Mattis, 1976). Measures of verbal and semantic abilities included the Boston Naming Test (Kaplan, Goodglass, \& Weintraub, 1982), the comprehension sub-test of the Western Aphasia Battery (Kertesz, 1982), the verbal fluency task for the letters F, A, and S, and the semantic fluency task for the 'animal' category (Benton, Hemsher, Varney, \& Spreen, 1983). Also, the FTD group completed the picture version of the Pyramids and Palms Trees Test, a non-verbal measure of semantic processing (Howard \& Patterson, 1992).

Verbal memory and learning were assessed with the California Verbal Learning Task (CVLT) (Delis, Kramer, Kaplan, \& Ober, 1987), except for one FTD patient (case 4) who completed the Hopkins Verbal Learning Test (Benedict, Schretlen, Groninger, \& Brandt, 1998). Visuo-spatial abilities were assessed with the Judgment of Line Orientation Test (Benton et al., 1983), and with the visual memory subtest from the Weschler Memory Scale-Revised (WMS-R), which included both immediate and delayed reproduction (Weschler, 1987).

Working memory was assessed by comparing backward and forward digit span tasks of the Weschler Memory ScaleRevised (WMS-R). The ability to switch mental sets was assessed by the ratio of Trail Making Test Part B to Part A
(Reitan \& Wolfson, 1993) and the Wisconsin Card Sorting Task (WCST) assessed categorization ability as well as set switching. One AD patient was unable to understand the instructions to the Trails B and one FTD patient (case 1) became frustrated with the WCST and walked out.

Neuropsychiatric testing included the Frontal Behavioral Inventory (Kertesz et al., 2000), the Neuropysychiatric Inventory area (Cummings et al., 1994), and the Cornell Scale for Depression in Dementia (Alexopoulos et al., 1988).

The Frontal Behavioral Inventory (FBI) is a standarized 24-item questionnaire that assesses the major behavioral changes characteristic of frontotemporal dementia, and has shown some reliability in discriminating FTD from other dementias. The questionnaire was completed with assistance of the patient's caretaker. Data for three AD patients were unavailable. As expected, the FTD group showed higher scores than the AD group, $t(10): 3.0, p<0.01$. Five FTD patients and one AD patient had a score higher than the cut-off of 30 . The only FTD patient with a score below cut-off was Case 1, but in this case the guardian was a colleague who did not have intimate knowledge of the patient's behavior.

The Neuropsychiatry Inventory (NPI) is a validated, widely used, semi-structured interview by the clinician with the caregiver to assess 12 behavioral domains, including delusions, hallucinations, agitation, depression, anxiety, euphoria, apathy, disinhibition, irritability, aberrant motor behavior, night-time behavior, and appetite disturbance (Cummings et al., 1994). The inventory takes into account both frequency (on a scale $0-4$ ) and severity (on a scale $0-3$ ) of each disorder for a maximum of 12 points in each. Data were gathered for all patients except one AD patient and one FTD patient (case 1) for whom caregiver reports were unavailable (see Table 1). Four of five FTD patients had abnormally high scores in the NPI, particularly in disinhibition, apathy, changes in appetite, and aberrant motor behavior. In contrast, only two of eight AD patients showed increased scores in the NPI.

The Cornell Scale for Depression in Dementia is a clinician-led checklist of depressive symptoms obtained from interviews with the patient and the caregiver. Data were collected for all patients except one AD patient, who showed signs of mild depression in the Geriatric Depression Scale (Burke et al., 1991Burke, Roccaforte, \& Wengel, 1991), and one FTD patient (RH, case 1) who had a history of depression treated with SSRIs. Four of five FTD patients had high scores, consistent with the overlap between FTD and depression in terms of apathy, changes in appetite, and irritability. The other FTD patient exhibited euphoria (case 4). Three of eight AD patients had scores higher than $25 \%$, suggesting probable depression, and two had high scores in the FBI.

\section{References}

Adolphs, R., Damasio, H., Tranel, D., Cooper, G., \& Damasio, A. R. (2000). A role for somatosensory cortices in the visual recognition of 
emotion as revealed by three-dimensional lesion mapping. The Journal of Neuroscience, 20(7), 2683-2690.

Adolphs, R., Tranel, D., Damasio, H., \& Damasio, A. R. (1994). Impaired recognition of emotion in facial expressions following bilateral damage to the human amygdala. Nature, 372, 669-672.

Albert, M. S., Cohen, C., \& Koff, E. (1991). Perception of affect in patients with dementia of the Alzheimer type. Archives of Neurology, 48, 791-795.

Alexopoulos, G. S., Abrams, R. C., Young, R. C., \& Shamoian, C. A. (1988). Cornell Scale for Depression in Dementia. Biological Psychiatry, 23(3), 271-284.

Allison, T., Puce, A., \& McCarthy, G. (2000). Social perception from visual cues: The role of the STS region. Trends in Cognitive Science, 4(7), 267-278.

Anderson, A. K., Spencer, D. D., Fullbright, R. K., \& Phelps, E. A. (2000). Contribution of the anteromedial temporal lobes to the evaluation of facial emotion. Neuropsychology, 14, 526-536.

Asthana, H. S., Mandal, M. K., Khurana, H., \& Haque-Nizamie, S. (1998). Visuo-spatial and affect recognition deficit in depression. Journal of Affective Disorders, 48, 57-62.

Benedict, R. H. B., Schretlen, D., Groninger, L., \& Brandt, J. (1998). Hopkins verbal learning test-revised: Normative data and analysis of inter-form and test-retest reliability. The Clinical Neuropsychologist, 12(1), 43-55.

Benton, A. L., Hemsher, K. S., Varney, N., \& Spreen, O. (1983). Contributions to neuropsychological assessment: A clinical manual. Oxford: Oxford University Press (pp. 30-43).

Blair, R. J. R., Morris, J. S., Frith, C. D., Perret, D. I., \& Dolan, R. (1999). Dissociable neural responses to facial expressions of sadness and anger. Brain, 122, 883-893.

Boccardi, M., Pennanen, C., Laakso, M. P., Testa, C., Geroldi, C., Soininen, H., et al. (2002). Amygdaloid atrophy in frontotemporal dementia and Alzheimer's disease. Neuroscience Letters, 335, 139-143.

Bocti, C., Rockel, C., Roy, P., Gao, F. Q., \& Black, S. E. (2004). Differential topography of Alzheimer's disease and frontotemporal dementia: An MRI volumetric analysis from the Sunnybrook Dementia Study. Brain and Cognition, 54(2), 151-153.

Bowers, D., Blonder, L. X., Feinberg, T., \& Heilman, K. M. (1991). Differential impact of right and left hemisphere lesions on facial emotion and object imagery. Brain, 114, 2590-2609.

Bruce, V., \& Young, A. (1986). Understanding face recognition. British Journal of Psychology, 77, 305-327.

Burke, W. J., Roccaforte, W. H., \& Wengel, S. P. (1991). The short form of the Geriatric Depression Scale: A comparison with the 30-item form. Journal of Geriatric Psychiatry and Neurology, 4(3), 173-178.

Calder, A. J., Lawrence, A. D., \& Young, A. W. (2001). Neuropsychology of fear and loathing. Nature Reviews Neuroscience, 2, 352-363.

Callen, D. J. A., Black, S. E., Gao, F., Caldwell, C. B., \& Szalai, J. P. (2001). Beyond the hippocampus: MRI volumetry confirms widespread limbic atrophy in AD. Neurology, 57, 1669-1674.

Cummings, J. L., Mega, M., Gray, K., Rosenberg-Thompson, S., Carusi, D. A., \& Gornbein, J. (1994). The Neuropsychiatric Inventory: Comprehensive assessment of psychopathology in dementia. Neurology, 44, 2308-2314.

Damasio, A. R., Damasio, H., \& Van Hoesen, G. W. (1982). Prosopagnosia: Anatomic basis and behavioral mechanisms. Neurology, 33, 331-341.

Delis, D., Kramer, J., Kaplan, E., \& Ober, B. (1987). The California verbal learning test. New York: Psychological Corp.

Ekman, P., \& Friesen, W. V. (1975). Unmasking the face. Englewood Cliffs, NJ: Prentice-Hall.

Fernandez-Duque, D., \& Black, S. E. (2002). Impaired recognition of negative facial emotions in patients with Fronto-temporal dementia. In Poster presented at the annual conference of the Rotman Research Institute (pp. 25-26).

Fernandez-Duque, D., Hodges, S., \& Black, S. E. (2005). Empathic ability in frontotemporal dementia. Manuscript in preparation.
Folstein, M. F., Folstein, S. E., \& McHugh, P. R. (1975). 'Mini-mental state': A pratical method for grading the cognitive state of patients for the clinician. Journal of Psychiatric Research, 12, 185-198.

Gaebel, W., \& Wolwer, W. (1992). Facial expression and emotional face recognition in schizophrenia and depression. European Archives of Psychiatry \& Clinical Neuroscience, 242, 46-52.

Gessler, S., Cutting, J., Frith, C. D., \& Weinman, J. (1989). Schizophrenic inability to judge facial emotion: A controlled study. British Journal of Clinical Psychology, 28(Pt 1), 19-29.

Harmer, C. J., Thilo, K. V., Rothwell, J. C., \& Goodwin, G. M. (2001). Transcranial magnetic stimulation of medial-frontal cortex impairs the processing of angry facial expressions. Nature Neuroscience, 4(1), $17-18$.

Herzog, A. G., \& Kemper, T. L. (1980). Amygdaloid changes in aging and dementia. Archives of Neurology, 37, 625-629.

Hooper, M. W., \& Vogel, F. S. (1976). The limbic system in Alzheimer's disease. A neuropathological investigation. American Journal of Pathology, 85, 1-20.

Hornak, J., Rolls, E. T., \& Wade, D. (1996). Face and voice expression identification in patients with emotional and behavioral changes following ventral frontal lobe damage. Neuropsychologia, 34(4), 247-261.

Howard, D., \& Patterson, K. (Eds.). (1992). Pyramids and Palm Trees: A test of semantic access from pictures and words. Bury St. Edmunds, UK: Thames Valley Test Company.

Humphreys, G. W., Donnelly, N., \& Riddoch, M. J. (1993). Expression is computed separately from facial identity, and it is computed separately from moving and static faces: Neuropsychological evidence. Neuropsychologia, 31, 173-181.

Kanwisher, N., McDermott, J., \& Chun, M. M. (1997). The fusiform face area: A module in human extrastriate cortex specialized for face perception. Journal of Neuroscience, 17(11), 4302-4311.

Kaplan, E., Goodglass, H., \& Weintraub, S. (1982). Boston naming test. Philadelphia: Lea \& Febiger.

Keane, J., Calder, A. J., Hodges, J. R., \& Young, A. W. (2002). Face and emotion processing in frontal variant frontotemporal dementia. Neuropsychologia, 40, 655-665.

Kertesz, A. (1982). Western aphasia battery. New York: Grune \& Stratton.

Kertesz, A., Nadkarni, N., Davidson, W., \& Thomas, A. W. (2000). Teh frontal behavioral inventory in the differential diagnosis of frontotemporal dementia. Journal of International Neuropsychology, 6 , 460-468.

Lavenu, I., Pasquier, F., Lebert, F., Petit, H., \& Van der Linden, H. (1999). Perception of emotion in patients with fronto-temporal dementia and Alzheimer's disease. Alzheimer Disease and Associated Disorder, 5 , $32-40$.

LeDoux, J. E. (1996). The emotional brain: The mysterious underpinnings of emotional life. New York: Simon \& Schuster.

Mandal, M. K., \& Bhattacharya, B. B. (1985). Recognition of facial affect in depression. Perceptual and Motor Skills, 61, 13-14.

Mattis, S. (1976). Dementia rating scale. In R. Bellack \& B. Karasu (Eds.), Geriatric psychiatry. New York, NY: Grune \& Stratton.

McKhann, G. M., Albert, M. S., Grossman, M., Miller, B., Dickson, D., \& Trojanowski, J. Q. (2001). Clinical and pathological diagnosis of frontotemporal dementia: Report of the work group on frontotemporal dementia and Pick's disease. Archives of Neurology, 58, 1803-1809.

McKhann, G., Drachman, D., Folstein, M. F., Katzman, R., Price, D., \& Stadlan, E. M. (1984). Clinical diagnosis of Alzheimer's disease: Report of the NINCDS-ADRDA work group under the auspices of department of health and human services task force on Alzheimer's disease. Neurology, 34, 939-944.

Mychack, P., Rosen, H., \& Miller, B. L. (2001). Novel applications of social-personality measures to the study of dementia. Neurocase, 7 131-143.

Neary, D., Snowden, J. S., Gustafson, L., Passant, U., et al. (1998). Frontotemporal lobar degeneration: A consensus on clinical diagnostic criteria. Neurology, 51, 1546-1554. 
Neary, D., Snowden, J. S., Shields, R. A., Burjan, A. W., Northen, B., MacDermott, N., et al. (1987). Single photon emission tomography using 99mTc-HM-PAO in the investigation of dementia. Journal of Neurology, Neurosurgery, and Psychiatry, 50(9), 1101-1109.

Perry, R. J., Rosen, H. R., Kramer, J. H., Beer, J. S., Levenson, R. L., \& Miller, B. L. (2001). Hemispheric dominance for emotions, empathy, and social behaviour: Evidence from right and left handers with frontotemporal dementia. Neurocase, 7, 145-160.

Phillips, M. L., Young, A. W., Senior, C., Brammer, M., Calder, A. J., Bullmore, E. T., et al. (1997). A specific neural substrate for perceiving facial expressions of disgust. Nature, 389, 495-498.

Rapcsak, S. Z., Galper, S. R., Comer, J. F., Reminger, S. L., Nielsen, L., Kaszniak, A. W., et al. (2002). Fear recognition deficit after focal brain damage. Neurology, 54, 575-581.

Reitan, R., \& Wolfson, D. (1993). The Halstead-Reitan neuropsychological test battery: Theory and clinical interpretation. Tucson, AZ: Neuropsychology Press.

Rolls, E. T. (1999). The functions of the orbitofrontal cortex. Neurocase, $5,301-312$

Rosen, H. J., Perry, R. J., Murphy, J., Kramer, J. H., Mychack, P., Schuff, N., et al. (2002). Emotion comprehension in the temporal variant of frontotemporal dementia. Brain, 125, 2286-2295.
Russell, J. A. (1994). Is there universal recognition of emotion from facial expression? A review of cross-cultural studies. Psychological Bulletin, 115(1), 102-141

Tranel, D., Damasio, A. R., \& Damasio, H. (1988). Intact recognition of facial expression, gender, and age in patients with impaired recognition of face identity. Neurology, 38, 690-696.

Tsuchiya, K., Arima, K., Fukui, T., Kuroiwa, T., Haga, C., Iratini, S., et al. (1999). Distribution of basal ganglia lesions in Pick's disease with Pick bodies: A topographic neuropathological study of eight autopsy cases. Neuropathology, (19), 370-379.

Tsuchiya, K., \& Kosaka, K. (1990). Neuropathological study of the amygdala in presenile Alzheimer's disease. Journal of Neurological Sciences, 100, 165-173.

Weschler, D. A. (1987). Weschler memory scale: Revised. San Antonio, TX: Psychological Corporation.

Whalen, P. J., Rauch, S. L., Etcoff, N. L., McInerney, S. C., Lee, M. B., \& Jenike, M. A. (1998). Masked presentations of emotional facial expressions modulate amygdala activity without explicit knowledge. Journal of Neuroscience, 18(1), 411-418.

Young, A. W., Aggleton, J. P., Hellawell, D., Johnson, M., Broks, P., \& Hanley, J. R. (1993). Face processing impairments after amygdalotomy. Brain, 118, 15-24. 